\title{
Bright Triplet Self-Trapped Excitons to Dopant Energy Transfer in Halide Double-Perovskite Nanocrystals
}

Muyu Conga,b, Qingkai Zhang ${ }^{f}$, Bin Yang ${ }^{a, b^{*}}, J u n s h e n g ~ C h e n^{a}$, Jie Xiao ${ }^{b, e}$, Daoyuan Zheng ${ }^{c}$, Tiancheng Zheng $^{\mathrm{b}, \mathrm{d}}$, Ruiling Zhang ${ }^{\mathrm{c}}$, Guangyan Qing ${ }^{\mathrm{e}}$, Chunfeng Zhang ${ }^{\dagger}$ and Ke-li Han ${ }^{\mathrm{a}, \mathrm{b}, \mathrm{c}^{*}}$

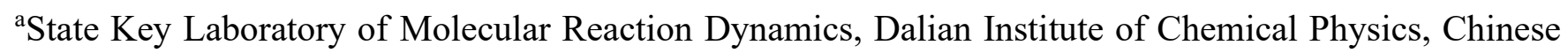
Academy of Science, Dalian 116023, P. R. China.

${ }^{\mathrm{b}}$ University of the Chinese Academy of sciences, Beijing 100049, P. R. China.

${ }^{\mathrm{c} I n s t i t u t e}$ of Molecular Sciences and Engineering, Shandong University, Qingdao 266237, P. R. China.

${ }^{\mathrm{d}}$ Key Laboratory of Chemical Lasers, Dalian Institute of Chemical Physics, Chinese Academy of Science, Dalian 116023, P. R. China.

${ }^{\mathrm{e} C A S}$ Key Laboratory of Separation Science of Analytical Chemistry, Dalian Institute of Chemical Physics, Chinese Academy of Science, Dalian 116023, P. R. China.

fSchool of Physics, Nanjing University, Nanjing 210093, P. R. China.

Email: yangbin@dicp.ac.cn

klhan@dicp.ac.cn 


\section{Materials}

\subsection{Materials}

In(OAc) 3 (99.99\%, Alfa Aesar), $\mathrm{Sb}(\mathrm{OAc})_{3}$ (99\%, Alfa Aesar), Cs(OAc) (99.99\%, Aladdin), Mn(OAc) $)_{2}$ (99.99\%, Alfa Aesar), K(OAc) (Anhydrous, Amresco), octadecene (90\%, Alfa Aesar), n-Hexane(98+\%, Aladdin), trimethysl chlorosilane (TMSCl, 98+\%, Alfa Aesar), oleic acid (OA, 90\%, Alfa Aesar), and oleylamine (OAm, 80-90\%, Aladdin). All materials were used as received without further purification.

\subsection{Synthesis of double perovskite NCs}

$0.71 \mathrm{mmol} \mathrm{Cs}(\mathrm{OAc})(136 \mathrm{mg}), 0.45 \mathrm{mmol} \mathrm{K}(\mathrm{OAc})(44 \mathrm{mg})$, and $0.45 \mathrm{mmol} \mathrm{In}(\mathrm{OAc})_{3}(130 \mathrm{mg})$ were dissolved in $10 \mathrm{~mL}$ octadecene. Then $2.8 \mathrm{~mL}$ oleic acid, and $0.7 \mathrm{~mL}$ oleylamine were added and this mixture solution was heated to $110{ }^{\circ} \mathrm{C}$ under vacuum for $1 \mathrm{~h}$. Subsequently, the reaction mixture was heated to $130{ }^{\circ} \mathrm{C}$ under a nitrogen atmosphere, and $0.4 \mathrm{ml}$ of $\mathrm{TMSCl}$ was injected swiftly. After $30 \mathrm{sec}$, the reaction mixture was cooled in an ice-water bath to room temperature. The reaction mixture was then centrifuged at $8000 \mathrm{rpm}$ for $10 \mathrm{~min}$. The supernatant was discarded, and the precipitate was dissolved into $5 \mathrm{ml} \mathrm{n}$-Hexane. The mixture was centrifuged at $8000 \mathrm{rpm}$ for $10 \mathrm{~min}$ again. The colloidal supernatant was collected for use.

The synthesis of $\mathrm{Cs}_{2} \mathrm{KIn}_{(1-\mathrm{x})} \mathrm{Sb}_{\mathrm{x}} \mathrm{Cl}_{6}(\mathrm{x}=0.07,0.1,0.2,0.3,0.4,0.5)$ NCs were performed in the similar methods, while $0.71 \mathrm{mmol} \mathrm{Cs}(\mathrm{OAc}), 0.45 \mathrm{mmol} \mathrm{K}(\mathrm{OAc}), 0.45^{*}(1-\mathrm{x}) \mathrm{mmol} \mathrm{In}(\mathrm{OAc})_{3}$ and $0.45^{*} \mathrm{x}$ mmol $\mathrm{Sb}(\mathrm{OAc})_{3}$ were used to form the precursor. For $\mathrm{Cs}_{2} \mathrm{~K}_{(1-2 \mathrm{y})} \mathrm{Mn}_{\mathrm{y}} \mathrm{In}_{0.9} \mathrm{Sb}_{0.1} \mathrm{Cl}_{6} \mathrm{NCs}$, the precursor were formed with $0.71 \mathrm{mmol} \mathrm{Cs}(\mathrm{OAc}), 0.45^{*}(1-2 \mathrm{y}) \mathrm{mmol} \mathrm{K}(\mathrm{OAc}), 0.4 \mathrm{mmol} \mathrm{In}(\mathrm{OAc})_{3}, 0.05 \mathrm{mmol} \mathrm{Sb}(\mathrm{OAc})_{3}$ and $0.45^{*} \mathrm{y} \mathrm{mmol} \mathrm{Mn}(\mathrm{OAc})_{2}$.

The $\mathrm{Sb}^{3+}$ doped $\mathrm{Cs}_{2} \mathrm{KInCl}_{6} \mathrm{NCs}$ with varying sizes were synthesized with the same mixture solution. The precursor was pumped vacuum at $110^{\circ} \mathrm{C}$. After $1 \mathrm{~h}$, the reaction temperature was changed to 90,110 , $130,150,170$, and $190^{\circ} \mathrm{C}$, respectively. Subsequently, $0.4 \mathrm{ml}$ of TMSCl was injected swiftly. After 0.5 , $0.5,0.5,1,3$, and 5 min reaction, respectively, the mixture cooled in an ice-water bath.

\section{Measurement and characterization}

\subsection{XRD measurements}

Powder X-ray diffraction (XRD) was performed on an X'pert PRO diffractometer equipped with $\mathrm{Cu} \mathrm{K}_{\alpha}$ $\mathrm{X}$-ray $(\lambda=1.54186 \AA)$ tubes. The colloidal NCs were drop coated on a glass substrate and heated at $50^{\circ} \mathrm{C}$ to prepare the thin films of DPs.

\subsection{TEM measurements}

The transmission electron microscopy (TEM) measurements were performed by using the JEM-2100. The colloidal solution was directly used for size distribution measurements.

\subsection{Analysis of element}

The inductively coupled plasma optical emission spectrometer (ICP-OES) 7300DV was used to analysis the content of $\mathrm{Sb} / \mathrm{Mn}$ alloyed double perovskite NCs. The double perovskite NCs were dissolved into nitric acid. The $\mathrm{Sb}$ content was calculated by the equation $\mathrm{Sb} \%=\mathrm{C}_{\mathrm{Sb}} /\left(\mathrm{C}_{\mathrm{Sb}}+\mathrm{C}_{\mathrm{In}}\right)$, and the Mn content was calculated by the equation $\mathrm{Mn} \%=\mathrm{C}_{\mathrm{Mn}} /\left(\mathrm{C}_{\mathrm{Mn}}+\mathrm{C}_{\mathrm{K}} / 2\right)$.

\subsection{PL spectroscopy measurements}

The steady-state PL spectroscopy, temperature-dependent PL, time-resolved PL spectra were recorded using a FLS1000 Edinburgh Instruments spectrofluorometer.

\subsection{Transient absorption}


TA experiments were carried out using a home-made femtosecond pump-probe setup. A Ti:sapphire femtosecond laser (Hurricane, Spectra- Physics) source was used to generate Laser pulses (800 nm, $50 \mathrm{fs}$ pulse length, $1 \mathrm{kHz}$ repetition rate). The laser wavelength was changed by using an optical parametric amplifier. We used the super-continuum generation from a thin $\mathrm{CaF}_{2}$ plate as the probe light. The mutual polarization between pump and probe beams was set to the magic angle $\left(54.7^{\circ}\right)$ by placing a Berek compensator in the pump beam. No sign of degradation could be observed during the long time of scanning. The temperature dependent TA measurements were performed by uniting the TA measurement with the Optistat DN. The TA measurements were performed for the double perovskite colloidal NCs with excitation wavelength at $320 \mathrm{~nm}$ for all samples.

\subsection{Magneto-optical spectroscopic measurement.}

For magneto-optical spectroscopic measurement, the samples were placed with a Faraday configuration in the sample chamber of a superconducting magnet (Spectromag SM4000, Oxford). The sample was pumped by the light emitting diode (homemade) at $\sim 310 \mathrm{~nm}$. The photoluminescence signal was measured using a monochromator (Acton SP2500 Princeton Instruments) with a back-illuminated charge-coupled device (PIXIS: 100B Princeton Instruments).

\subsection{Calculation of Huang-Rhys factors.}

The Huang-Rhys factor (S) reflects how strongly coupling between excitons and phonons. The difference between free excitons and self-trapped excitons is the magnitude of the carrier-phonon coupling. For free excitons, as the carrier-phonon coupling is weak, the $\mathrm{S}$ value can be obtained based on O'Donnell-Chen's theory. ${ }^{1}$ The $\mathrm{S}$ value was calculated by fitting with the equation $\mathrm{S} 1$ based on the temperature dependence of the semiconductor bandgap:

$$
E_{g}(T)=E_{g}(0)-S\langle\hbar \omega\rangle\left[\operatorname{coth}\left(\langle\hbar \omega\rangle / 2 k_{B} T\right)-1\right]
$$

where $\mathrm{E}_{\mathrm{g}}(0)$ is the bandgap at $\mathrm{T}=0 \mathrm{~K}, k_{B}$ is Boltzmann constant, and ( $\mathrm{h} \omega$ ) is an average phonon energy. This equation is usually used to calculate the free exciton with small $\mathrm{S}$ value, such as $\mathrm{CdSe}$ $(\mathrm{S}=1), \mathrm{CsPbBr}_{3}(\mathrm{~S}=3.2){ }^{2,3}$

For STEs, due to the strong coupling of excitons and phonons, the Huang-Rhys factor (S) can be obtained based on the theory of Toyozawa. ${ }^{4}$ This theory models the emission broadening resulting from electron-phonon interactions using a configuration coordinate model:

$$
F W H M=2.36 \sqrt{S}(\hbar \omega) \sqrt{\operatorname{coth}\left(\frac{(\hbar \omega)}{2 k_{B} T}\right)}
$$

which is due to the fact that a localized exciton cannot take the adiabatic approximation and apply the Franck-Condon principle to the transition. This equation has been widely used for STEs with high $\mathrm{S}$ value. Such as, $\mathrm{Cs}_{2} \mathrm{AgInCl}_{6}(\mathrm{~S}=38.7), \mathrm{Cs}_{3} \mathrm{Bi}_{2} \mathrm{I}_{9}(\mathrm{~S}=79.5){ }^{5}$

\subsection{Fabrication of WLEDs.}

We try the n-i-p structure to fabricate the electroluminescent devices, in which, the ZnO/PEIE (PEIE: polyethylenimine ethoxylated) was used as electron-transporting layer, the poly (9,9-dioctylfluorene-co-N-(4-butylphenyl) diphenylamine (TFB) was used as hole-transporting layer, and $\mathrm{Au}$ was used as electrodes. However, no obvious emission was observed from the fabricated device. This may be due to the misalignment of the energy levels considering the larger bandgap and low 
valence band energy level of DP2 NCs. And this inhibits the charge injection. Instead, we used DP2 NCs as the active phosphor material and fabricated a phosphor converted WLED based on DP2 NCs (Figure S16).

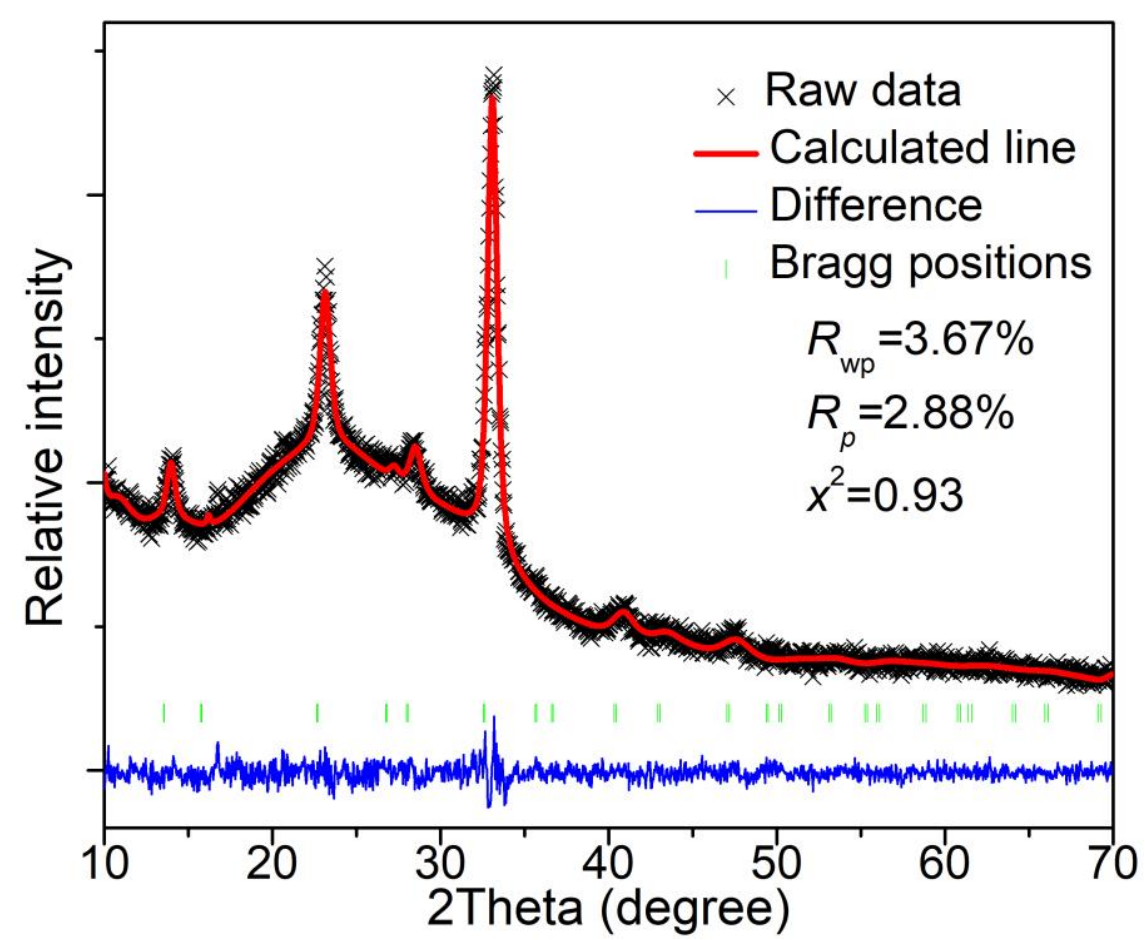

Figure S1. XRD refinement pattern of cubic $\mathrm{Cs}_{2} \mathrm{KInCl}_{6} \mathrm{NCs}$.

Table S1. Structural data for cubic $\mathrm{Cs}_{2} \mathrm{KInCl}_{6}$.

\begin{tabular}{|l|l|l|l|l|l|l|}
\hline Space Group & Atom & $\mathrm{X}$ & $\mathrm{Y}$ & $\mathrm{Z}$ & Occ. & Site \\
\hline \multirow{2}{*}{$\begin{array}{l}\text { Cubic } \\
\text { Fm3m } \\
\mathrm{a}=\mathrm{b}=\mathrm{c}=10.770 \AA\end{array}$} & $\mathrm{Cs}$ & 0.25 & 0.25 & 0.25 & 1.000 & $4 \mathrm{~b}$ \\
\cline { 2 - 7 } & $\mathrm{K}$ & 0 & 0.5 & 0.5 & 1.000 & $8 \mathrm{c}$ \\
\cline { 2 - 7 } & $\mathrm{In}$ & 0.5 & 0.5 & 0.5 & 1.000 & $24 \mathrm{e}$ \\
\cline { 2 - 7 } & $\mathrm{Cl}$ & 0.2724 & 0.5 & 0.5 & 1.000 & $4 \mathrm{a}$ \\
\hline
\end{tabular}

Table S2. ICP data of $10 \% \mathrm{Sb}^{3+}$ doped $\mathrm{Cs}_{2} \mathrm{KInCl}_{6} \mathrm{NCs}$ prepared at different temperature.

\begin{tabular}{c|c|c|c}
\hline $\begin{array}{c}\text { Synthesis temperature } \\
\left({ }^{\circ} \mathrm{C}\right)\end{array}$ & In $(\mathbf{m m o l} / \mathbf{m l})$ & Sb $(\boldsymbol{\mu m o l} / \mathbf{m l})$ & $\mathbf{S b}^{\mathbf{3 +}}$ content $(\%)$ \\
\hline 90 & 0.75 & 10.6 & 1.40 \\
\hline 110 & 0.79 & 6.9 & 0.86 \\
\hline 130 & 3.12 & 18.0 & 0.57 \\
\hline 150 & 0.22 & 9.2 & 0.41 \\
\hline 170 & 0.82 & 3.7 & 0.45 \\
\hline 190 & 1.34 & 4.5 & 0.34 \\
\hline
\end{tabular}


Table S3. ICP data of $\mathrm{Sb}^{3+}$ doped $\mathrm{Cs}_{2} \mathrm{KInCl}_{6} \mathrm{NCs}$ prepared at 170 and $190{ }^{\circ} \mathrm{C}$

\begin{tabular}{c|c|c|c|c}
\hline $\begin{array}{c}\text { Synthesis } \\
\text { temperature }\left({ }^{\circ} \mathrm{C}\right)\end{array}$ & $\begin{array}{c}\mathbf{S b}^{3+} \text { content in } \\
\text { precursor }\end{array}$ & In (mmol/ml) & Sb $(\boldsymbol{\mu m o l} / \mathbf{m l})$ & $\begin{array}{c}\mathbf{S b}^{3+} \text { content } \\
(\%)\end{array}$ \\
\hline \multirow{4}{*}{170} & $10 \%$ & 0.82 & 3.7 & 0.45 \\
\cline { 2 - 5 } & $20 \%$ & 0.57 & 8.9 & 1.53 \\
\cline { 2 - 5 } & $30 \%$ & 0.66 & 47.1 & 6.64 \\
\cline { 2 - 5 } & $40 \%$ & 0.80 & 47.0 & 19.72 \\
\hline \multirow{4}{*}{190} & $10 \%$ & 1.34 & 4.5 & 0.34 \\
\cline { 2 - 5 } & $20 \%$ & 0.16 & 2.2 & 1.36 \\
\cline { 2 - 5 } & $30 \%$ & 0.97 & 8.6 & 2.85 \\
\cline { 2 - 5 } & $40 \%$ & 0.81 & 136.8 & 14.4 \\
\hline
\end{tabular}

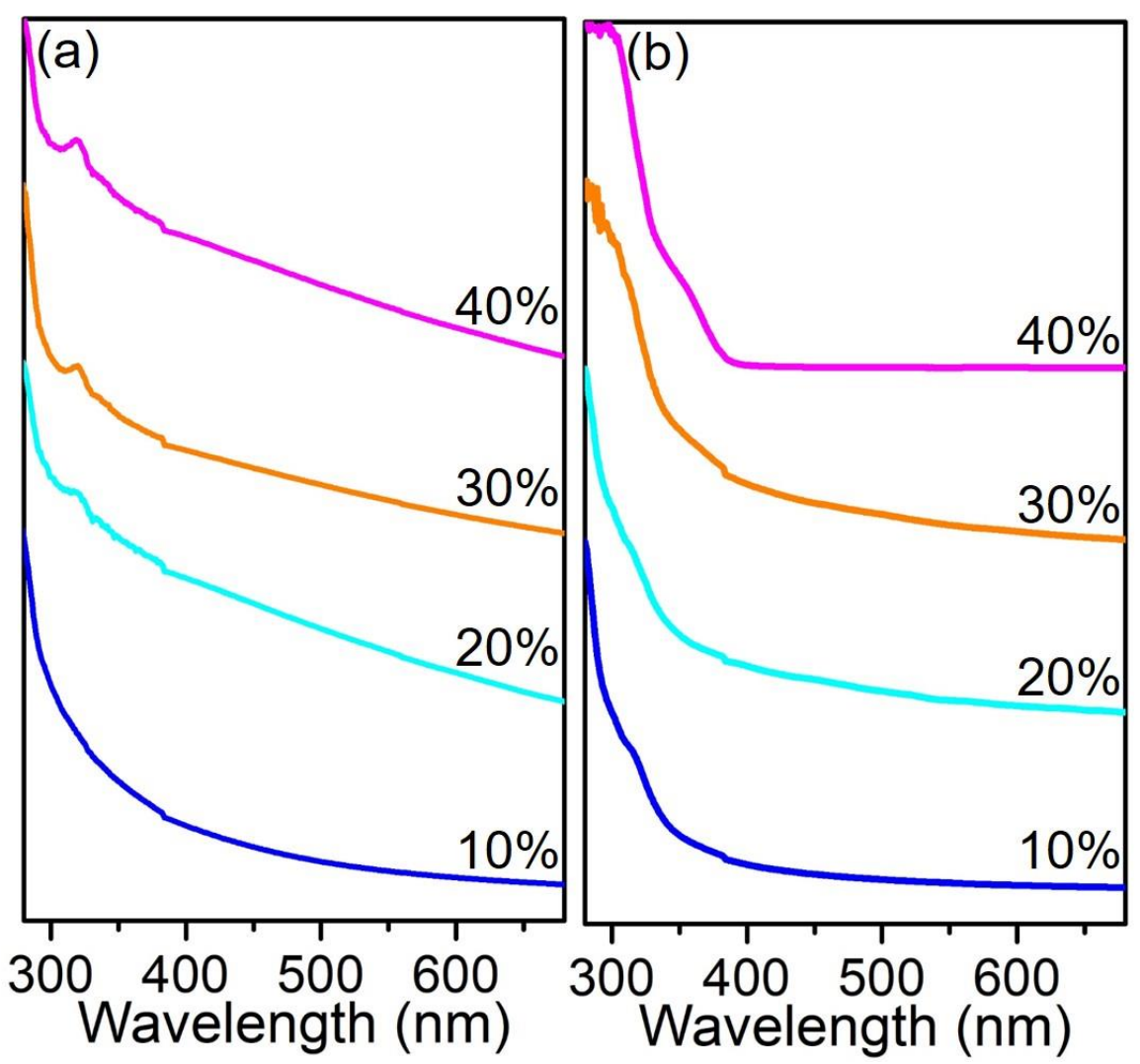

Figure S2. Absorption spectra of $\mathrm{Cs}_{2} \mathrm{KInCl}_{6} \mathrm{NCs}$ with different $\mathrm{Sb}$ content in precursor prepared at $170{ }^{\circ} \mathrm{C}(\mathrm{a})$ and $190{ }^{\circ} \mathrm{C}(\mathrm{b})$. The absorption peak at $320 \mathrm{~nm}$ is still not obvious at high Sb content. 


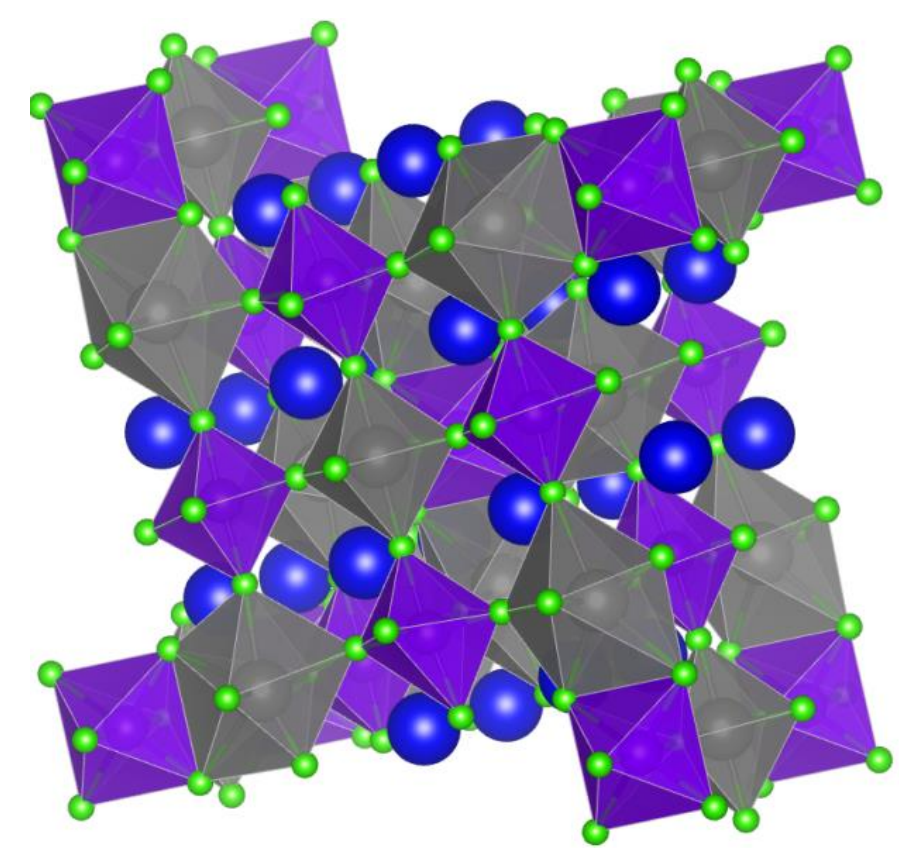

Figure S3. Crystal structure of tetragonal $\mathrm{Cs}_{2} \mathrm{KInCl}_{6}$ double perovskite.

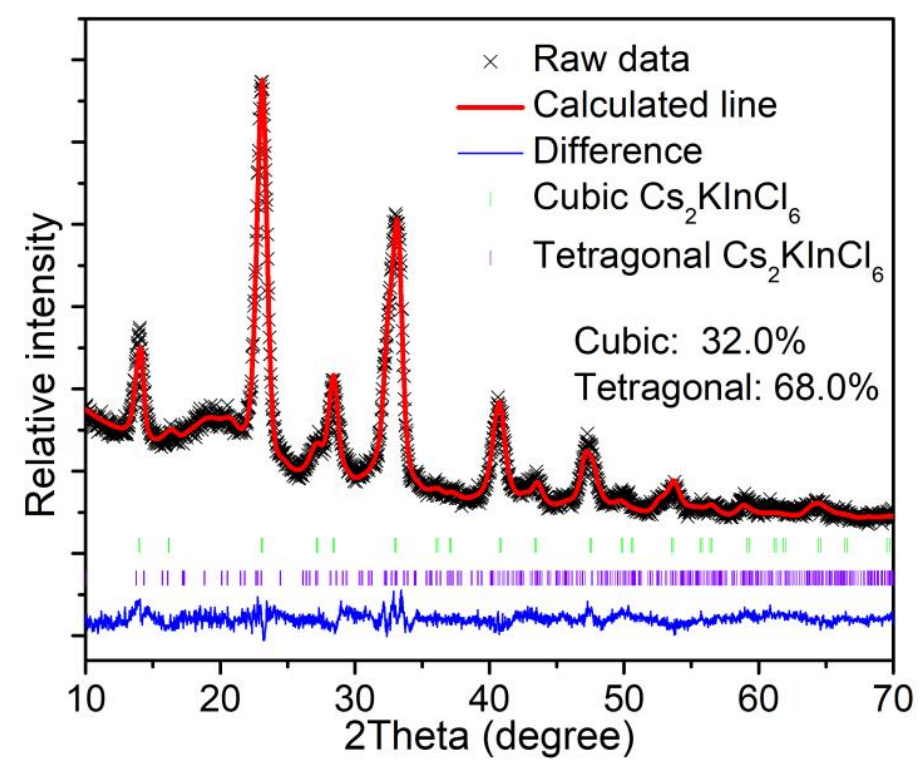

Figure S4. Calculation result of XRD data for $\mathrm{Sb}^{3+}$ doped $\mathrm{Cs}_{2} \mathrm{KInCl}_{6} \mathrm{NCs}$ prepared at $170{ }^{\circ} \mathrm{C}$. 


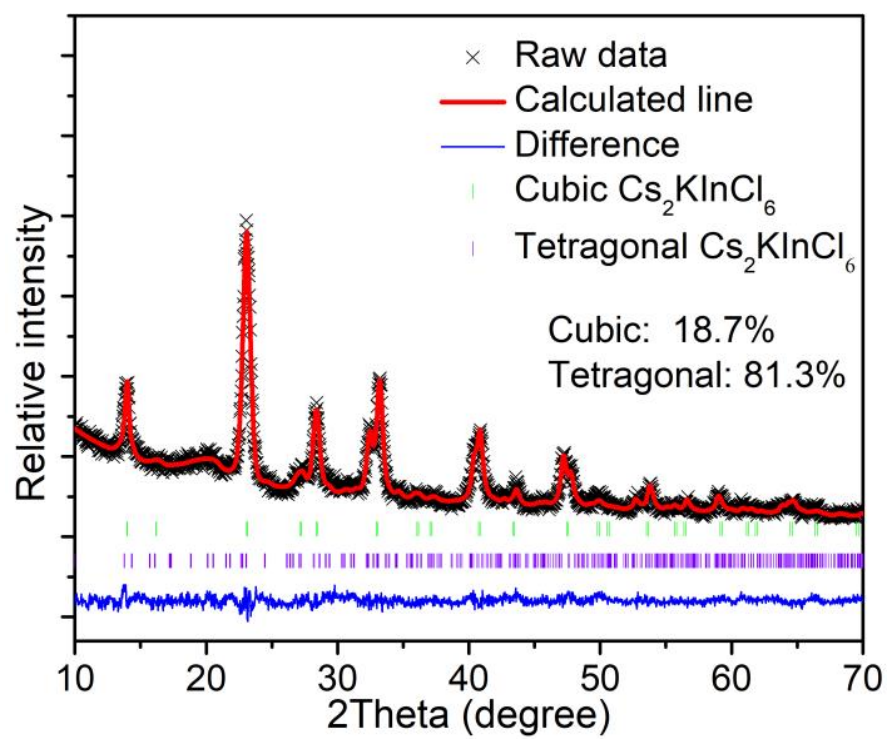

Figure S5. Calculation result of XRD data for $\mathrm{Sb}^{3+}$ doped $\mathrm{Cs}_{2} \mathrm{KInCl}_{6} \mathrm{NCs}$ prepared at $190{ }^{\circ} \mathrm{C}$.

Table S4. The FWHM values of PL for NCs prepared at different temperature.

\begin{tabular}{c|c|c|c|c|c|c}
\hline Temperature/ ${ }^{\circ} \mathrm{C}$ & 90 & 110 & 130 & 150 & 170 & 190 \\
\hline FWHM/nm & 116 & 115 & 114 & 112 & 116.5 & 145 \\
\hline FWHM/meV & 549 & 543 & 540 & 540 & 560 & 574 \\
\hline
\end{tabular}

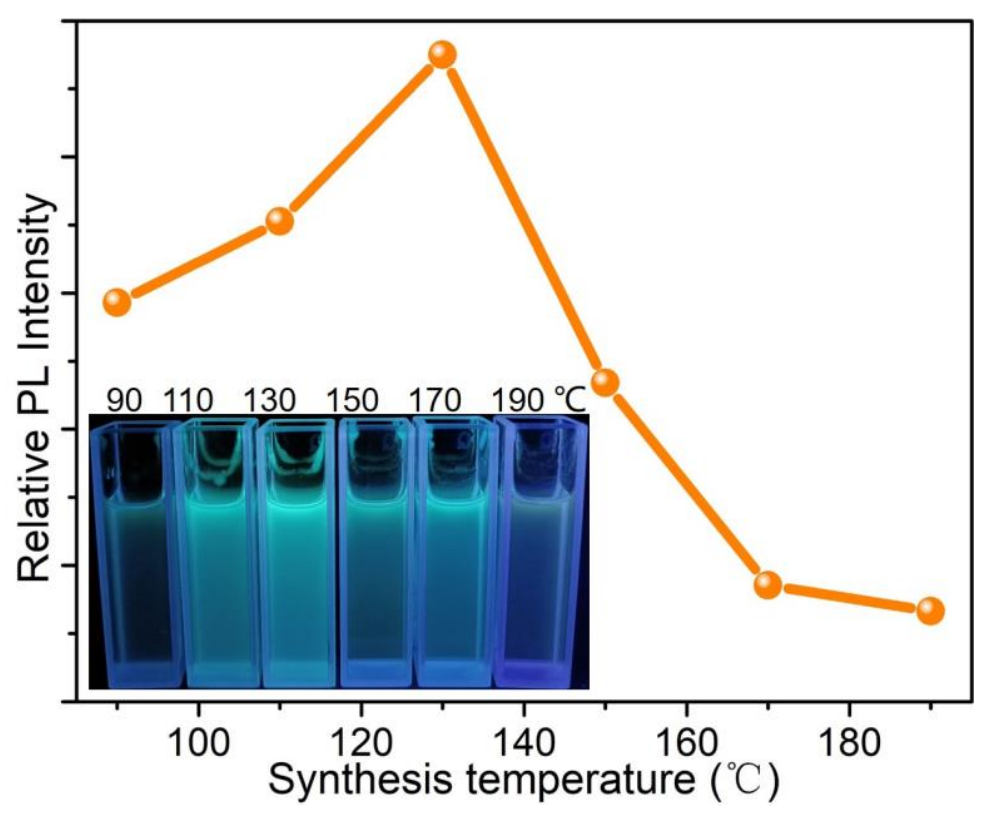

Figure S6. Relative PL intensity of $10 \% \mathrm{Sb}^{3+}$ doped $\mathrm{Cs}_{2} \mathrm{KInCl}_{6} \mathrm{NCs}$ synthesized at different temperature. Illustration: $10 \% \mathrm{Sb}^{3+}$ doped $\mathrm{Cs}_{2} \mathrm{KInCl}_{6} \mathrm{NCs}$ prepared at different temperatures under $254 \mathrm{~nm} \mathrm{UV}$ light. 
Table S5. ICP data of $\mathrm{Sb}^{3+}$ doped $\mathrm{Cs}_{2} \mathrm{KInCl}_{6} \mathrm{NCs}$ synthesized at $130^{\circ} \mathrm{C}$.

\begin{tabular}{c|c|c|c}
\hline $\mathbf{S b}^{\mathbf{3 +}}$ content in precursor & $\mathbf{I n}(\mathbf{m m o l} / \mathbf{m l})$ & $\mathbf{S b}(\boldsymbol{\mu \mathbf { m o l }} / \mathbf{m l})$ & $\mathbf{S b}^{\mathbf{3 +}}$ content $(\%)$ \\
\hline $7 \%$ & 1.49 & 2.7 & 0.18 \\
\hline $10 \%$ & 1.56 & 9.1 & 0.57 \\
\hline $20 \%$ & 1.09 & 14.9 & 1.36 \\
\hline $30 \%$ & 1.25 & 25.5 & 2.00 \\
\hline $40 \%$ & 0.88 & 31.6 & 3.45 \\
\hline $50 \%$ & 0.63 & 47.3 & 7.00 \\
\hline
\end{tabular}
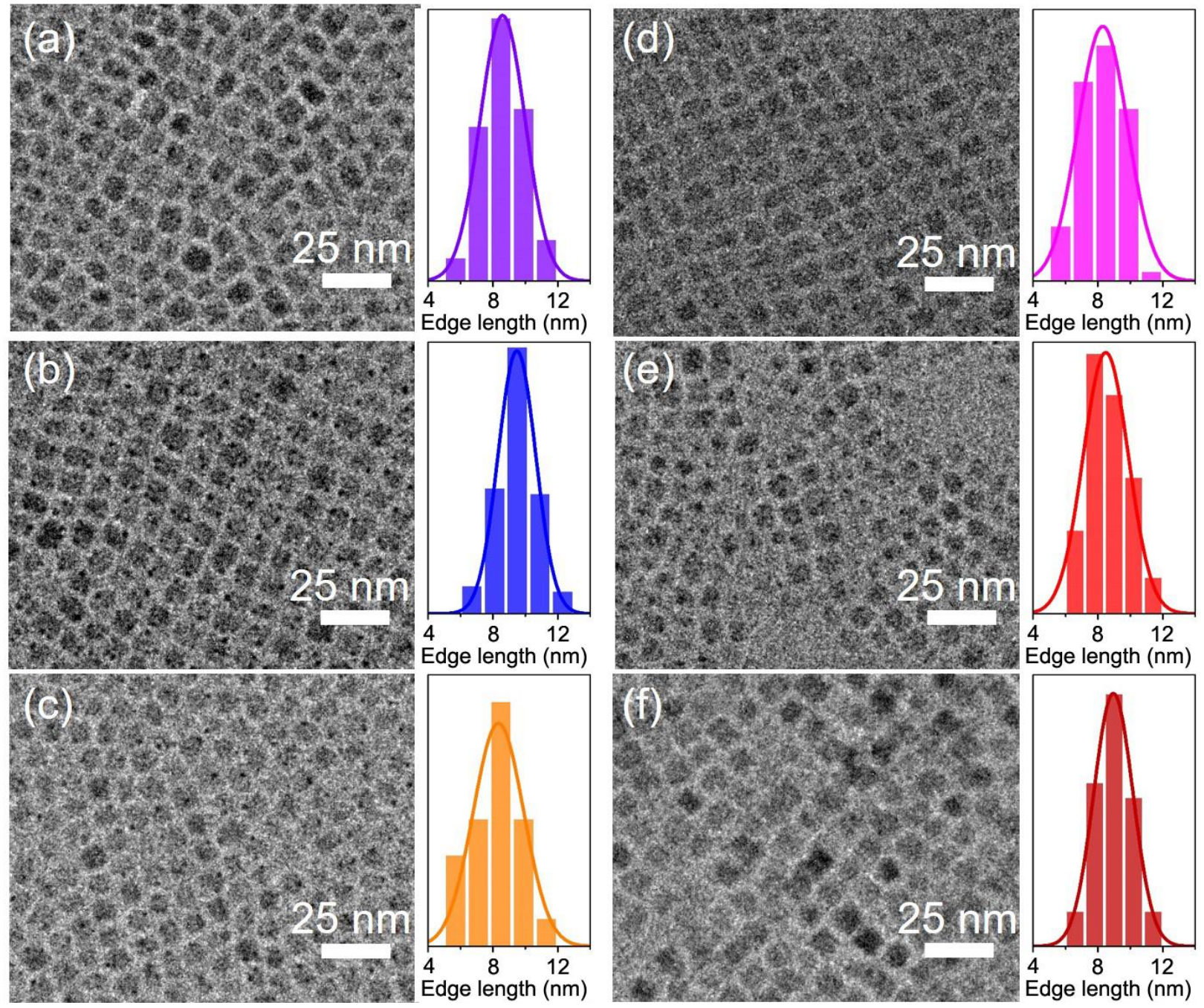

Figure S7. TEM images and their size distributions histograms of $\mathrm{Cs}_{2} \mathrm{KInCl}_{6} \mathrm{NCs}$ doped with $7 \%$ (a), $10 \%$ (b), $20 \%$ (c), $30 \%$ (d), $40 \%$ (e), and $50 \%$ (f) $\mathrm{Sb}^{3+}$, synthesized at $130{ }^{\circ} \mathrm{C}$. The edge size of these double perovskite NCs are 8.6 (7\%), 9.4 (10\%), $8.2(20 \%), 8.3(30 \%), 8.7(40 \%), 9.0(50 \%) \mathrm{nm}$, respectively. 

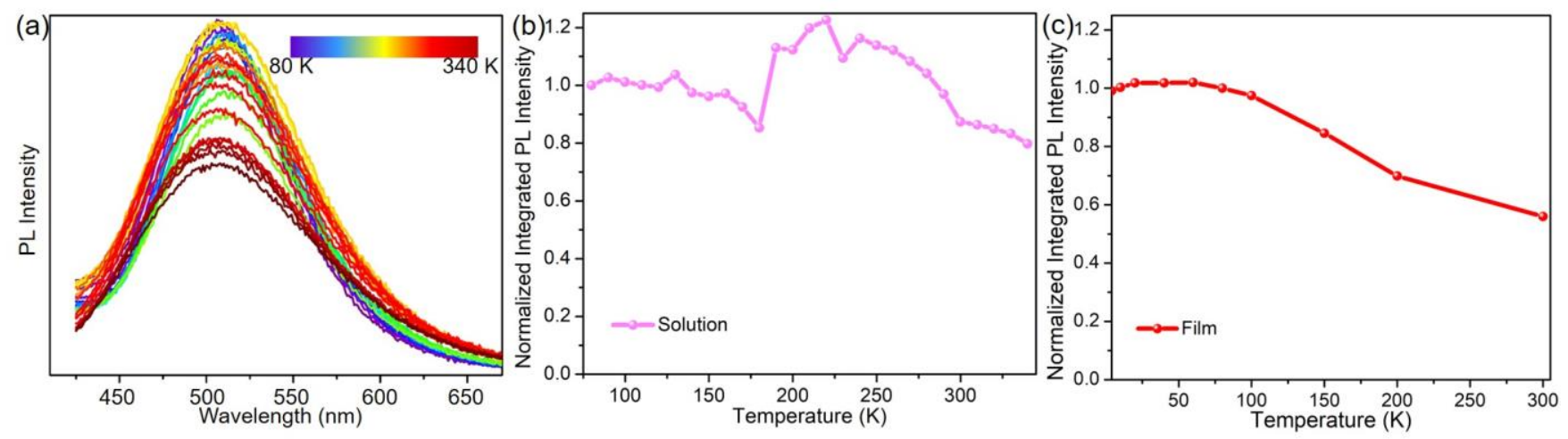

Figure S8. (a) Temperature dependent PL of DP1 NCs with temperature ranging from 80 to $340 \mathrm{~K}$. (b) Temperature dependent integrated PL intensity of DP1 NCs. The temperature dependent integrated PL intensity can be divided into two regions. First region: from 80 to $180 \mathrm{~K}$ (solid state). Second region: from $220 \mathrm{~K}$ to $340 \mathrm{~K}$ (colloidal state). The PL intensity decreased with increasing temperature in both regions. We also noted there is abrupt PL enhancement from around $180 \mathrm{~K}$ to $220 \mathrm{~K}$. This may be due to the melting of n-hexane (melting point: $178 \mathrm{~K}$ ), when the colloidal becomes frozen, the sample turns to cloudy, and which could increase the light scattering and decrease the PL intensity. We further measured the integrated PL intensity of solid thin films NCs from 4-300 K to eliminate the influence of solvent. The integrated PL of thin films show slightly decreased PL with increasing temperature without abrupt change in PL intensity (c).

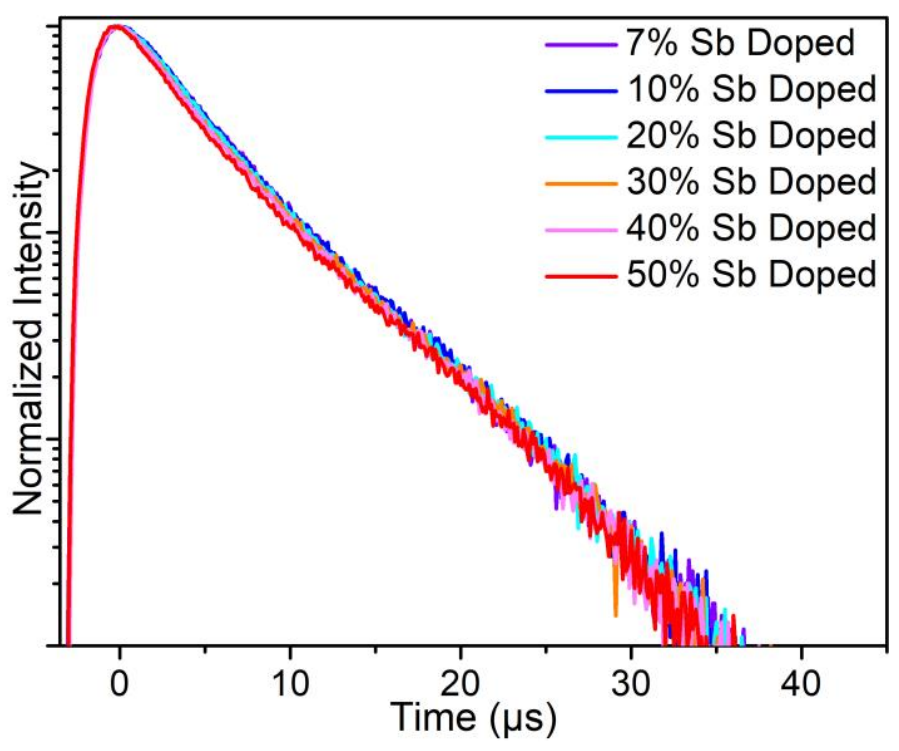

Figure S9. TR-PL kinetics of $\mathrm{Cs}_{2} \mathrm{KInCl}_{6}$ doped with different content $\mathrm{Sb}^{3+}$. 

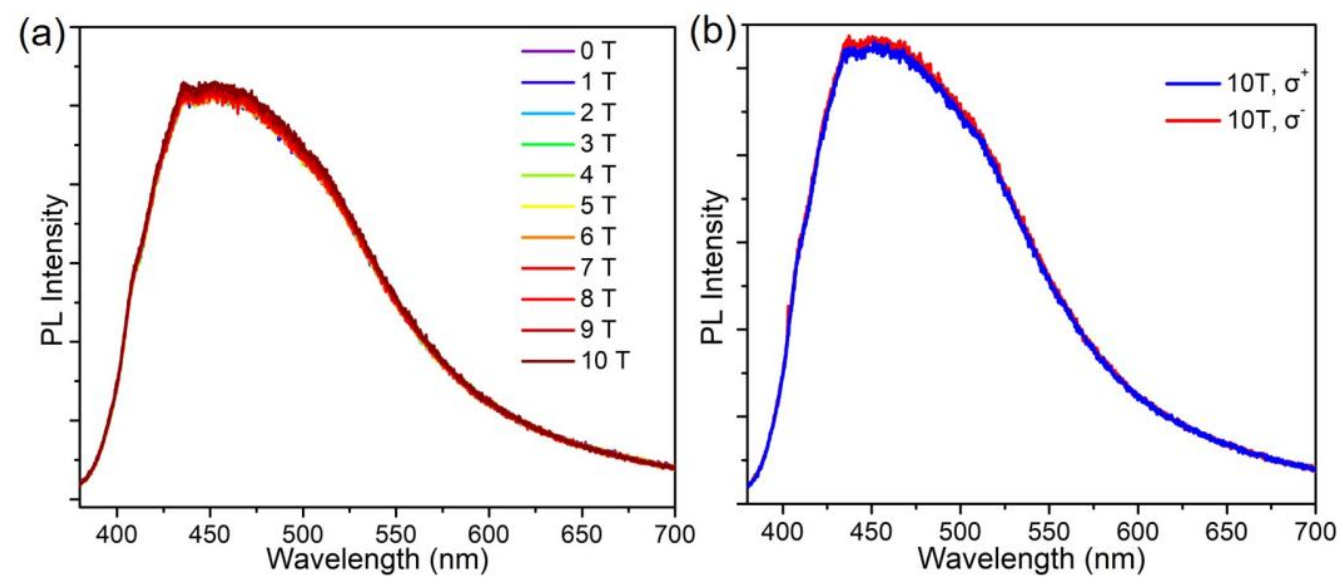

Figure S10. (a) PL spectra with magnetic field changes from 0 to $10 \mathrm{~T}$ (at 4K). (b) Circular polarization PL under magnetic field of $10 \mathrm{~T}$ (at $4 \mathrm{~K})$. The PL of right circularly polarized luminescence $\left(\sigma^{+}\right)$is identical to that of left circularly polarized luminescence $\left(\sigma^{-}\right)$.
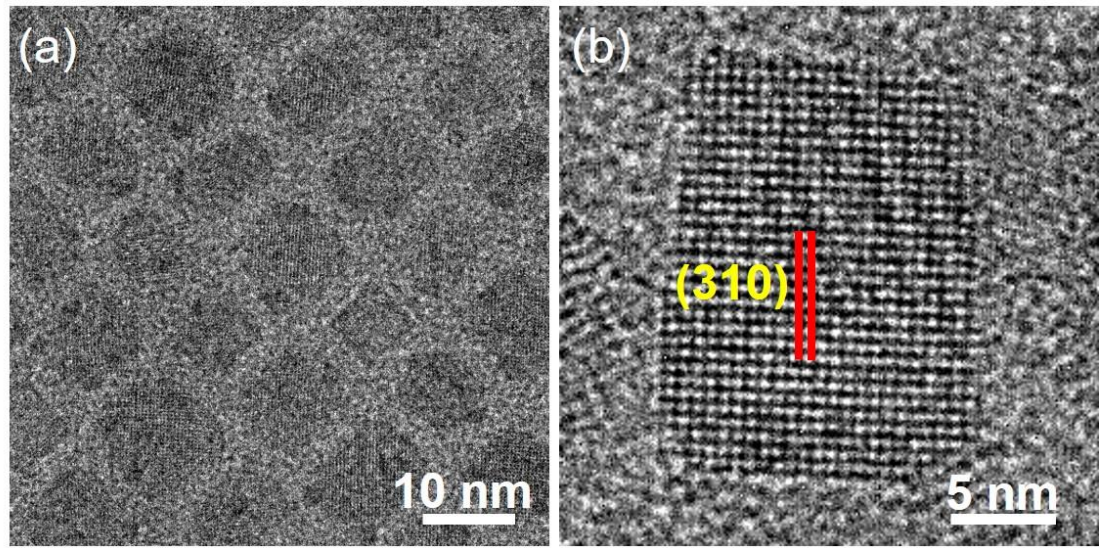

Figure S11. TEM (a) and HRTEM (b) images of DP2 NCs.

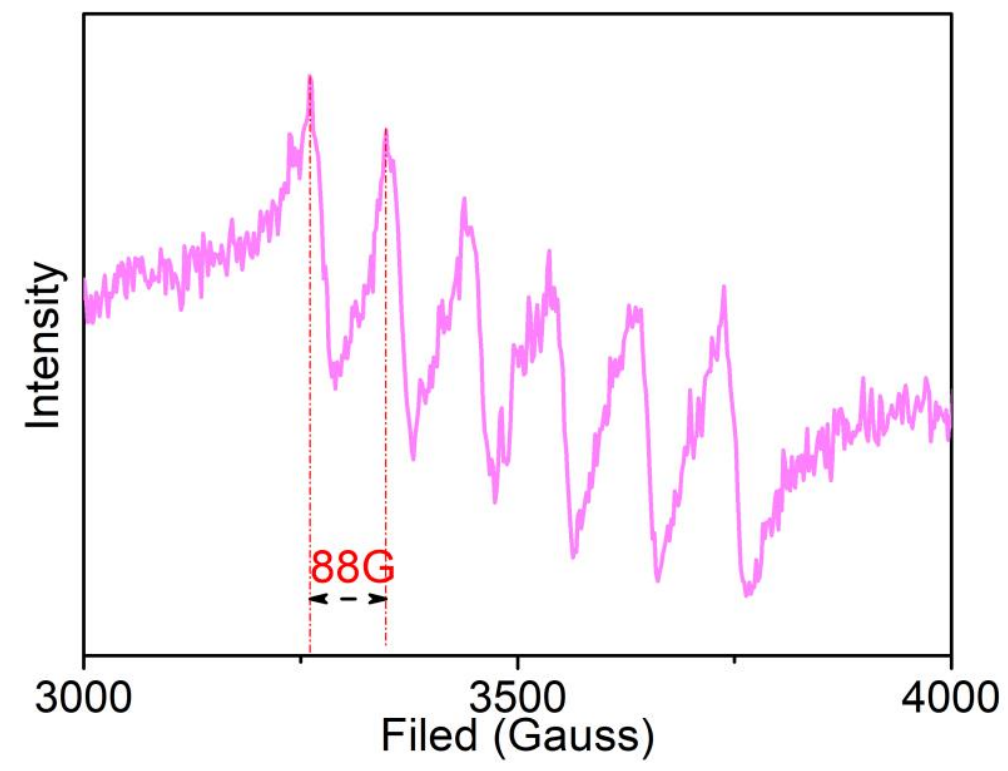

Figure S12. Room temperature X-band EPR spectrum of DP2 NCs. 
Table S6. ICP data of $\mathrm{Mn}^{2+}$ doped DP1 NCs

\begin{tabular}{c|c|c|c}
\hline $\mathbf{M n}^{2+}$ content in precursor & $\mathbf{K}(\mathbf{m m o l} / \mathbf{m l})$ & $\mathbf{M n}(\boldsymbol{\mu m o l} / \mathbf{m l})$ & $\mathbf{M n}^{2+}$ content $(\%)$ \\
\hline $1 \%$ & 1.36 & 10.4 & 1.51 \\
\hline $3 \%$ & 1.37 & 27.2 & 3.84 \\
\hline $5 \%$ & 0.68 & 15.7 & 4.41 \\
\hline $10 \%$ & 0.73 & 42.1 & 10.34 \\
\hline $20 \%$ & 0.68 & 63.1 & 15.65 \\
\hline
\end{tabular}

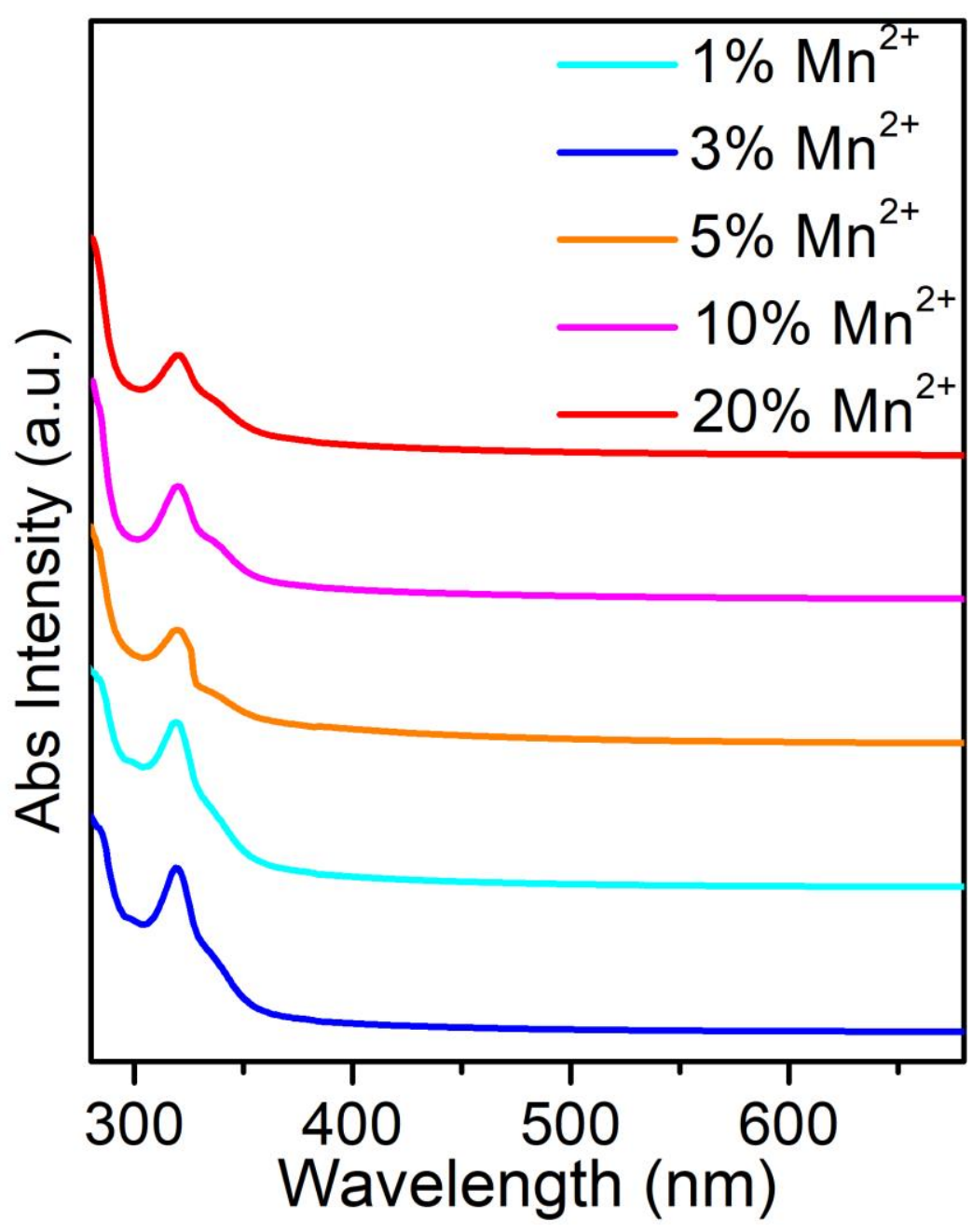

Figure S13. Absorption spectrum of $\mathrm{Mn}^{2+}$ alloyed DP1 NCs. 


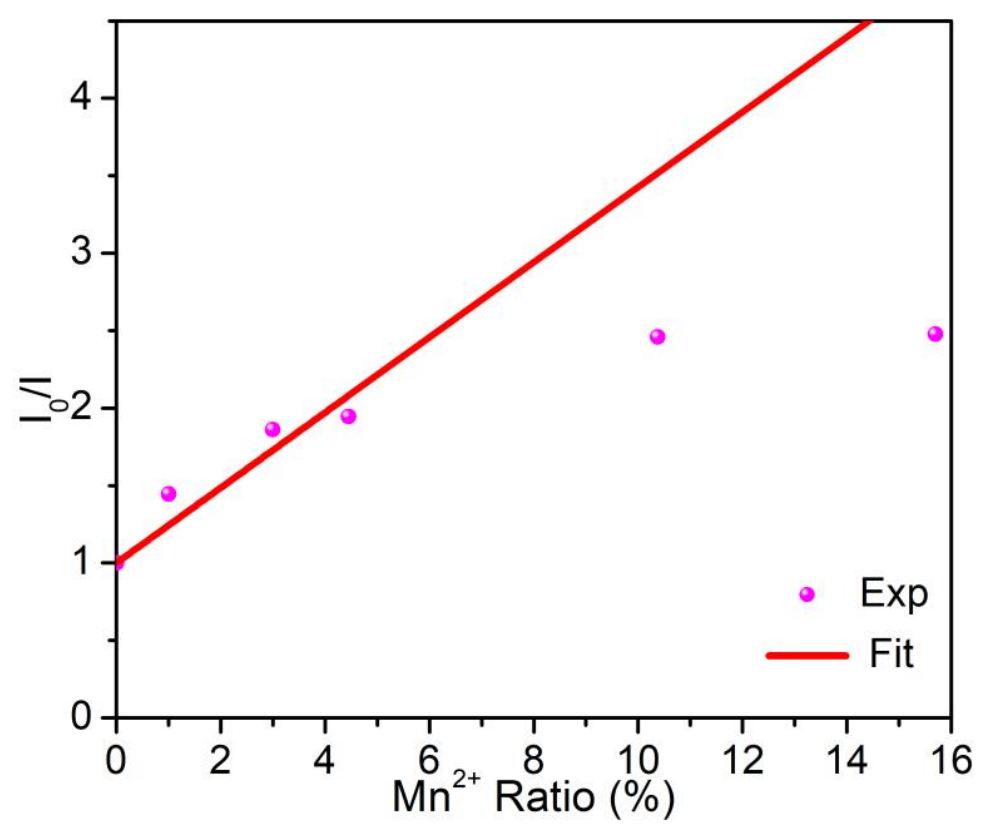

Figure S14. Relationship between PL intensity and $\mathrm{Mn}^{2+}$ concentration fitted with a Stern-Volmer equation $\mathrm{I}_{0} / \mathrm{I}=1+\mathrm{K}_{\mathrm{SV}}[\mathrm{Mn}]$, where $\mathrm{I}_{0}$ and $\mathrm{I}$ represent the sum of PL intensity around 510 and $630 \mathrm{~nm}$ and the PL intensity around $510 \mathrm{~nm}$, respectively. $\left[\mathrm{Mn}\right.$ ] is the ratio of $\mathrm{Mn}^{2+}$. With the increase of $\mathrm{Mn}$ content from $5 \%$ to $10 \%, \mathrm{I}_{0} / \mathrm{I}$ deviated from the fitting curve based on Stern-Volmer equation (Figure S14), which describes the expected PL enhancement of peak 2 relative to peak 1 with increasing Mn content. $^{30}$ The deviation between experimental results and the theoretical value indicates that there should be other nonradiative processes that quenching the $\mathrm{Mn}^{2+}$ based emission. Concentration quenching is always observed in inorganic and hybrid Mn based phosphors, in which the excitation energy transfer from an initially absorbing $\mathrm{Mn}$ ions to another identical ions, and finally reach to a quenching site (such as crystal defects). ${ }^{6,7}$ It have been observed that the PLQY of organo Mn based halide clusters have a direct relation with the closest Mn-Mn distance - a longer Mn-Mn distance leads to the higher PLQY. ${ }^{7}$ At low Mn content $(<5 \%)$, the $\left[\mathrm{MnCl}_{6}\right]$ octahedron may be isolated from each other, which hinders the energy transfer and PL quenching (concentration quenching). However, with the increasing of Mn content (>5\%), there would existence $\left[\mathrm{MnCl}_{6}\right]$ octahedron clusters, which promotes the energy transfer and PL quenching.

Table S7. Lifetime of DP1 NCs doped with different Mn content.

\begin{tabular}{c|c|c|c|c}
\hline $\begin{array}{c}\mathbf{M n}^{2+} \text { content in } \\
\text { precursor }\end{array}$ & A1 $(\%)$ & Time (ms) & A2 (\%) & Time (ms) \\
\hline $1 \%$ & 39.57 & 3.36 & 60.43 & 26.75 \\
\hline $3 \%$ & 49.62 & 2.37 & 50.38 & 25.23 \\
\hline $5 \%$ & 50.67 & 2.09 & 49.33 & 21.20 \\
\hline $10 \%$ & 60.80 & 1.81 & 39.20 & 17.46 \\
\hline $20 \%$ & 73.98 & 0.79 & 26.02 & 11.55 \\
\hline
\end{tabular}




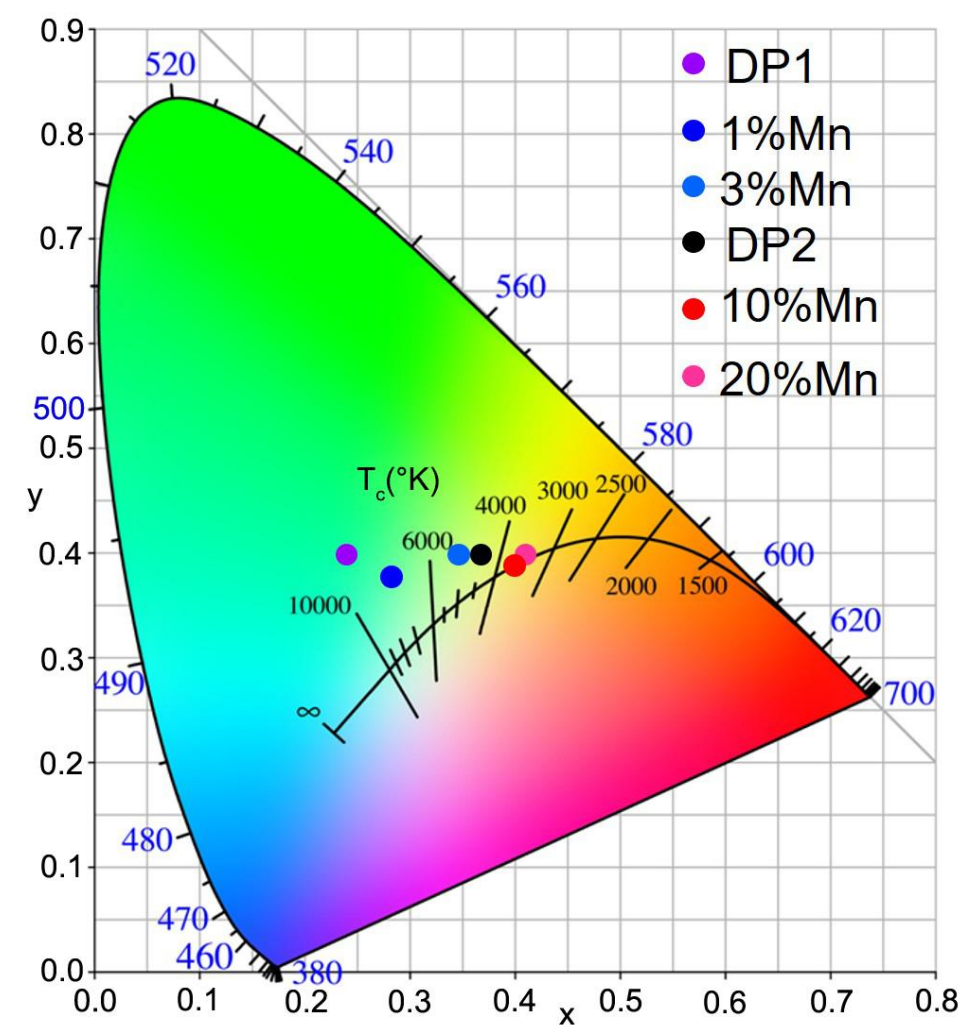

Figure S15. CIE images of double perovskite NCs.

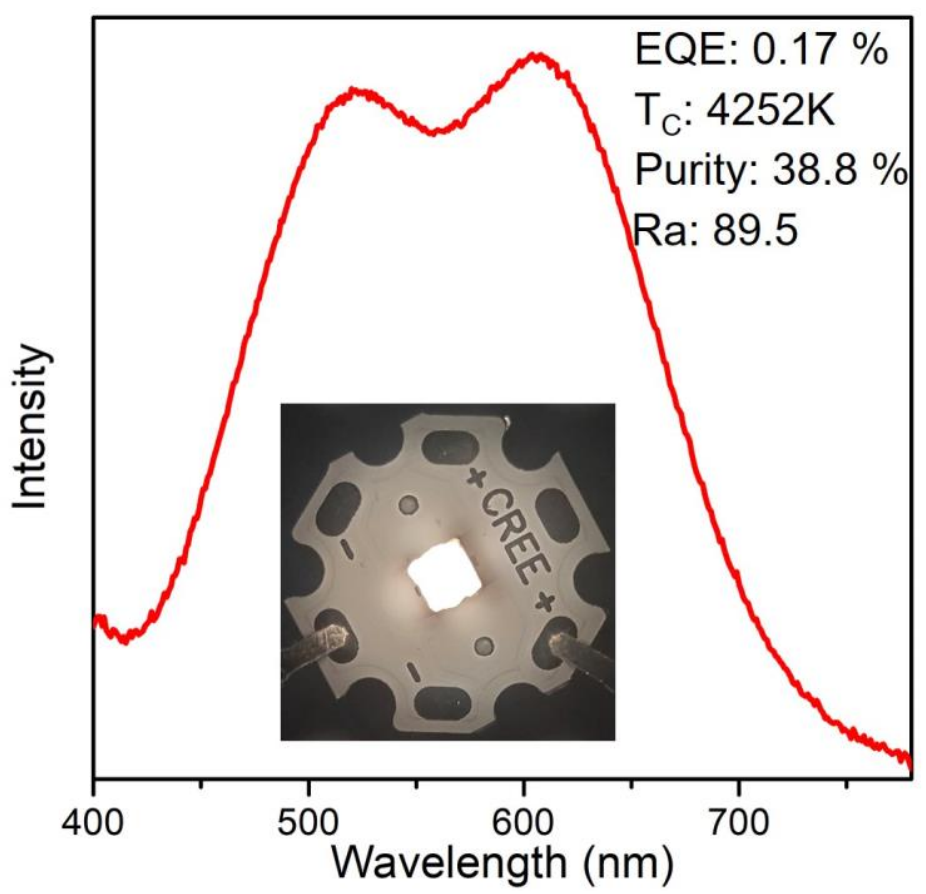

Figure S16. Electroluminescence spectra of WLED chip based on DP2 NCs. In which, the DP2 NCs were coated on UV-LED chips $(310 \mathrm{~nm})$ at an applied current of $120 \mathrm{~mA}$. The insert is the photograph of the fabricated WLED chip. 


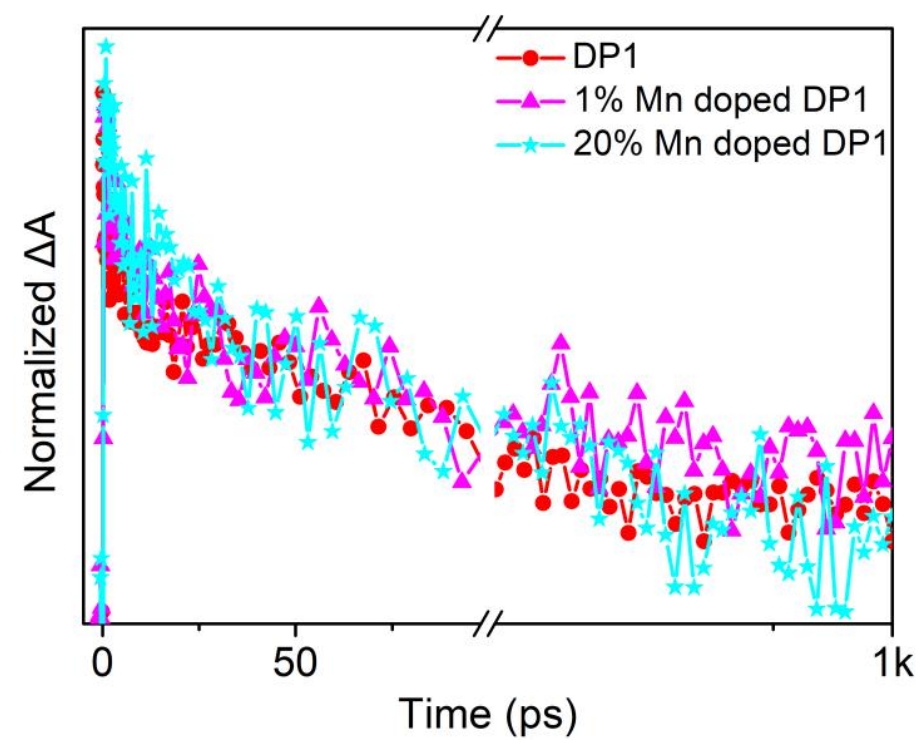

Figure S17. PIA decay for different content $\mathrm{Mn}^{2+}$ alloyed DP1 NCs.
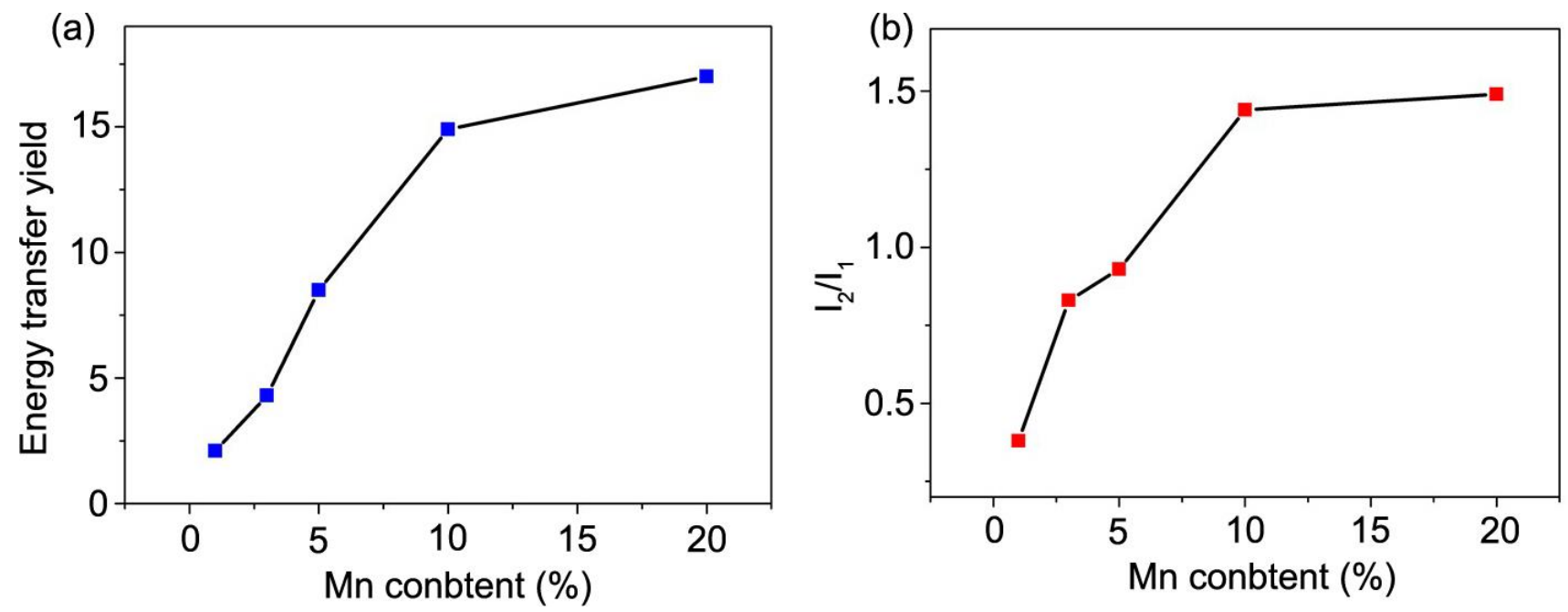

Figure S18. (a) Mn content-dependent energy transfer yield. (b) Mn content dependent $I_{2} / I_{1}\left(I_{2}\right.$ : intensity of PL peak $2 ; \mathrm{I}_{1}$ : intensity of PL peak 1$)$. The energy transfer yield is calculated based on $\left(\mathrm{k}_{\mathrm{DP} 1-\mathrm{Mn}}-\mathrm{k}_{\mathrm{DP} 1}\right) /$ $\mathrm{k}_{\mathrm{DP} 1-\mathrm{Mn}}$, where $\mathrm{k}_{\mathrm{DP} 1-\mathrm{Mn}}$ is the recombination rate of peak 1 for $\mathrm{Mn}$ doped DP1, while $\mathrm{k}_{\mathrm{DP} 1}$ is the recombination rate of peak 1 for DP1. 

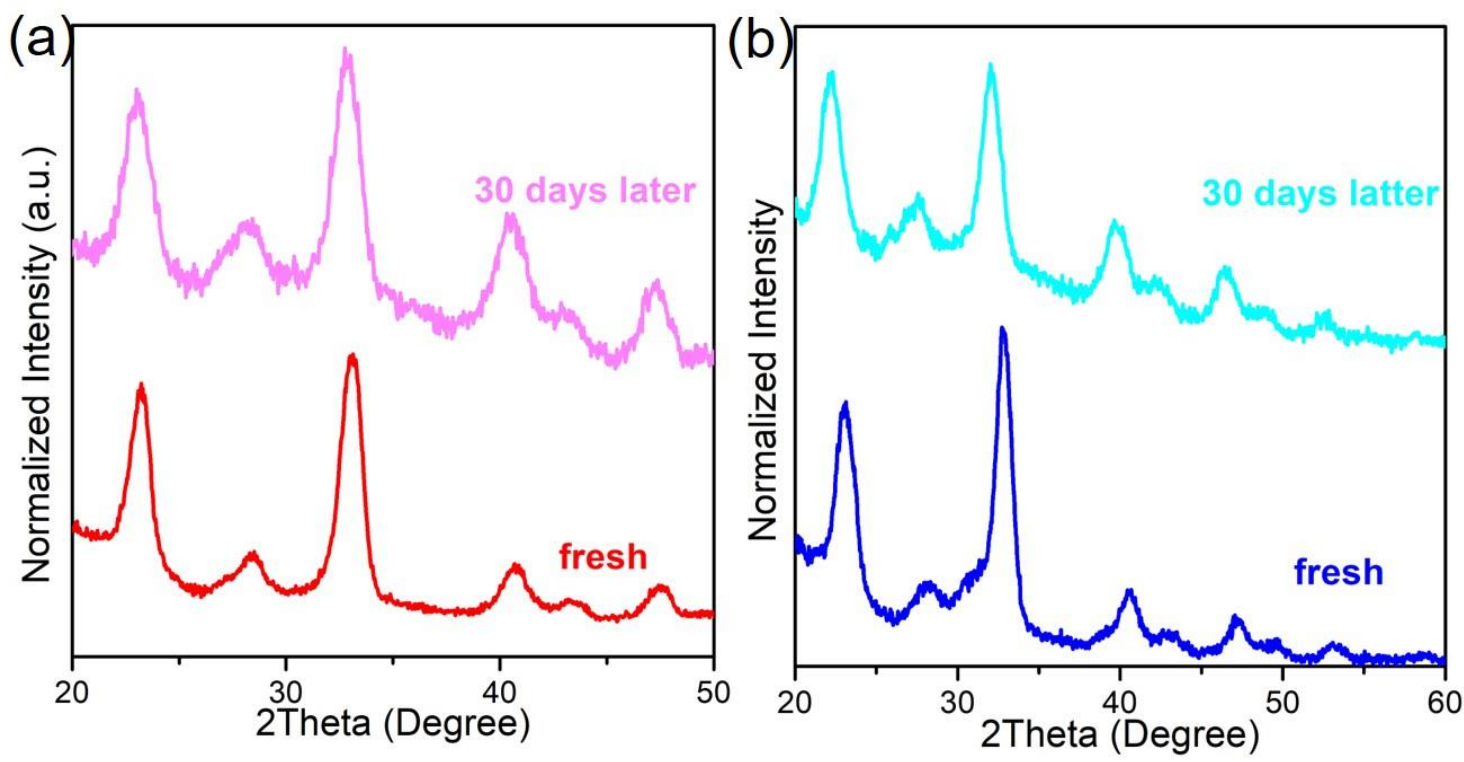

Figure S19. (a) XRD patterns of fresh DP1 NCs and DP1 NCs after being exposed to air for 30 days. (b) XRD patterns of fresh DP2 NCs and DP2 NCs after being exposed to air for 30 days.
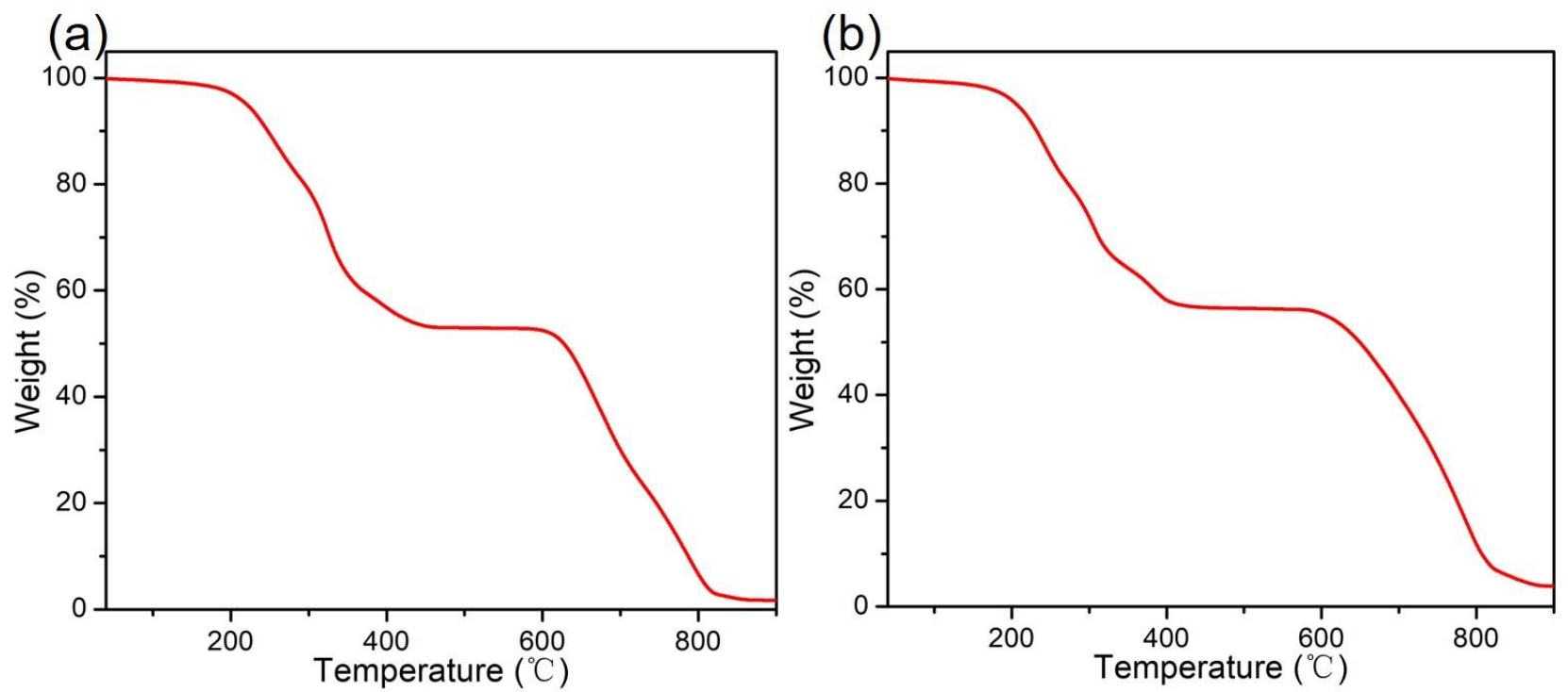

Figure S20. Thermogravimetric analysis (TGA) thermogram of DP1 (a) and DP2 (b) NCs. 


\section{Reference:}

(1) O’Donnell, K. P.; Chen, X. Temperature dependence of semiconductor band gaps. Appl. Phys. Lett. 1991, 58, 2924-2926.

(2) Türck, V.; Rodt, S.; Stier, O.; Heitz, R.; Engelhardt, R.; Pohl, U. W.; Bimberg, D.; Steingrüberm, R. Effect of random field fluctuations on excitonic transitions of individual CdSe quantum dots. Phys. Rev. B 2000, 61, 9944.

(3) Lao, X.; Yang, Z.; Su, Z.; Wang, Z.; Ye, H.; Wang, M.; Yao, X.; Xu, S. Luminescence and thermal behaviors of free and trapped excitons in cesium lead halide perovskite nanosheets. Nanoscale 2018, 10, 9949-9956.

(4) Toyazawa, Y. Theory of Lin-Shapes of the Exciton Absorption Bands. Prog. Theor. Phys. 1958, 20, 53-80.

(5) McCall, K. M.; Stoumpos, C. C.; Kostina, S. S.; Kanatzidis, M. G.; Wessels, B. W. Strong Electron-Phonon Coupling and Self-Trapped Excitons in the Defect Halide Perovskites $\mathrm{A}_{3} \mathrm{M}_{2} \mathrm{I}_{9}(\mathrm{~A}=\mathrm{Cs}$, $\mathrm{Rb} ; \mathrm{M}=\mathrm{Bi}, \mathrm{Sb})$. Chem. Mater. 2017, 29, 4129-4145.

(6) Dexter, D. L.; Schulman, J. H. Theory of Concentration Quenching in Inorganic Phosphors. J. Chem. Phys. 1954, 22, 1063-1070.

(7) Mao, L.; Guo, P.; Wang, S.; Cheetham, A. K.; Seshadri, R. Design Principles for Enhancing Photoluminescence Quantum Yield in Hybrid Manganese Bromides. J. Am. Chem. Soc. 2020, 142, 13582-13589. 